\title{
Apparent transducer non-reciprocity in an ultrasonic flow meter
}

\author{
Jan van Deventer *, Jerker Delsing \\ EISLAB, Lulea University of Technology, Luleå S-97187, Sweden
}

\begin{abstract}
This paper investigates the effects of non-identical ultrasonic transducers on reciprocity and zero-flow calibration in transit time flow meters. According to the theorem of reciprocity, there should not be any difference between the up- and downstream acoustic times of flight in a zero-flow situation. This would thus eliminate zero-flow estimation drifts. The flow meter is modeled as a one dimensional system with equivalent electrical circuits and simulated with simulation program with integrated circuits emphasis. The work shows that variations between the two transducers cause false estimates of flow and indicate which parameters have the largest influence. It indicates that reciprocity holds only for identical transducers. (c) 2002 Elsevier Science B.V. All rights reserved.
\end{abstract}

Keywords: Ultrasonic flow meter; Simulation; SPICE; Reciprocity

\section{Introduction}

A transit time ultrasonic flow meter estimates the flow velocity by transmitting acoustic pulses up- and downstream with respect to the direction of the flow. From the time of the pulses' flight, the direction of the flow, its velocity and the velocity of sound in the fluid can be estimated [1]. Fig. 1 shows a cross-section of such a meter.

When the fluid is not moving, the up and down times of flight should be the same. If they are not, the meter detects it as a false flow. Hemp [2] shows that this error vanishes when applying the theorem of reciprocity. Willatzen [3] recently published a tutorial on transducer modeling with a general theory and applications to ultrasound reciprocal systems. He also relies on the fact that the transmitter and the receiver are identical for the system to be reciprocal.

To literally quote Pierce [4], reciprocity refers to the situation for which a magnitude associated with an "effect" at a point is unchanged when the location of "cause" and "point of observation" are interchanged. The current work shows that reciprocity does hold when the two transducers are identical. It additionally shows that some measurement violations exist with small variations of the transducers. These variations are due to the manufacturing process of the transducers. In the

\footnotetext{
${ }^{*}$ Corresponding author.

URL: http://www.eislab.sm.luth.se/.
}

next section, we present the simulation tool used to evaluate small changes of properties or dimensions in each of the layers of one of the two transducers as well as its wiring.

\section{Simulation tool}

We use the electronics simulation package named simulation program with integrated circuits emphasis (SPICE) to simulate an ultrasonic system. The analogous system has the passive layers of the flow meter modeled by transmission lines [5]. The active elements, the piezoelectric disks, use an electrically equivalent circuit developed by Leach [6].

Fig. 2 shows the schematics of the flow meter's equivalent electrical circuit. The electrical pulses are generated by a pulsed voltage sources or pulsers (pSource). The pulses rise from 0 to $24 \mathrm{~V}$ in $100 \mathrm{~ns}$ and falls half of a resonant period later at the same rate. The resonant period is the time of one complete cycle of the resonant frequency of the disk. Following the pulsed excitation, the voltage sources are disconnected via a switch from the piezoelectric element (Venable, top of Fig. 2). This provides a higher impedance to measure any incoming acoustic signal (done at output of the pulsers). Two $30 \mathrm{~cm}$ long $50 \Omega$ coaxial cables connect the pulsers to the piezoelectric disks of the transducers. The modeled piezoelectric disks are $0.5 \mathrm{~mm}$ thick Pz27 


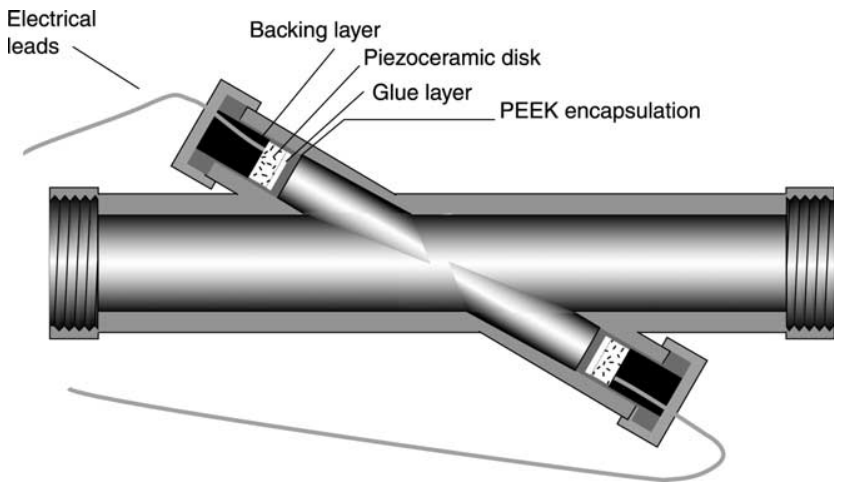

Fig. 1. Cut away of a transit time ultrasonic flow meter.

disks from Ferroperm, Denmark. They have a $10 \mathrm{~mm}$ diameter. The piezoelectric disks are allowed to discharge through a $100 \Omega$ resistor in the pulsers. The piezoelectric disks are backed by a tungsten filled epoxy with a $5 \mathrm{MPa} / \mathrm{m}$ acoustic impedance. The front of the piezoelectric disks are glued with an epoxy to the polyetheretherketone (PEEK) encapsulation. The epoxy layer is one-quarter of a wavelength thick while the PEEK layer is three-quarters of a wavelength thick. In between the two facing transducers, the liquid medium is $20 \mathrm{~mm}$ long. Fig. 3 shows the pulses received by the two transducers where the second transducer has a $10 \%$ thinner PEEK thickness.

\section{Simulation results}

The advantage of using simulations is that the influence of one parameter at the time can be studied. The individually varied parameters are the thickness of the PEEK encapsulation, the thickness of the glue layer, the acoustic impedance of the backing layer, the length of the coaxial cable, the thickness and permittivity of the piezoelectric disk. The results are given in Fig. 4 and tabulated in Table 1. The parameters of only the second transducer are varied from $-10 \%$ to $+10 \%$ of their nominal values. This range covers the manufacturer's electrical tolerances of the piezoelectric disks. The results of the simulations are the time difference between the up- and downstream times of flight. These times of flight are defined from the start of the excitation to the first (negative) zero crossing of the received pulse.

The values in Table 1 obtained for the variations of the PEEK thickness are numerical residuals. Forcing the simulations time steps to a smaller value will reduce them. However, this is at the cost of much longer simulations.

As opposed to Hemp [2], Johansson [7] points out that the time delay and amplitude variations caused by the coaxial cable should not be overlooked. With the permittivity being $-10 \%$ on one of the transducers, the time difference is $1.5 \mathrm{~ns}$ without the coaxial cable and $1.9 \mathrm{~ns}$ with. Thus, variations in the characteristics of the cable do introduce zero-flow errors and promote any others present.

Variations in the associated electronics are not studied here. Although tolerances in electronic components do create a difference in time of flights, those are nowadays minimized due to the use of application-specific integrated circuits (ASICs). Matching between transistors can be achieved with a high degree of precision within a silicon chip [8].

It could be easily argued that the measurement method is at fault. A cross-correlation method of the two received signals will also introduce errors, as two non-identical transducers will have different responses. The real issue is that non-identical transducers will have different responses to inputs.

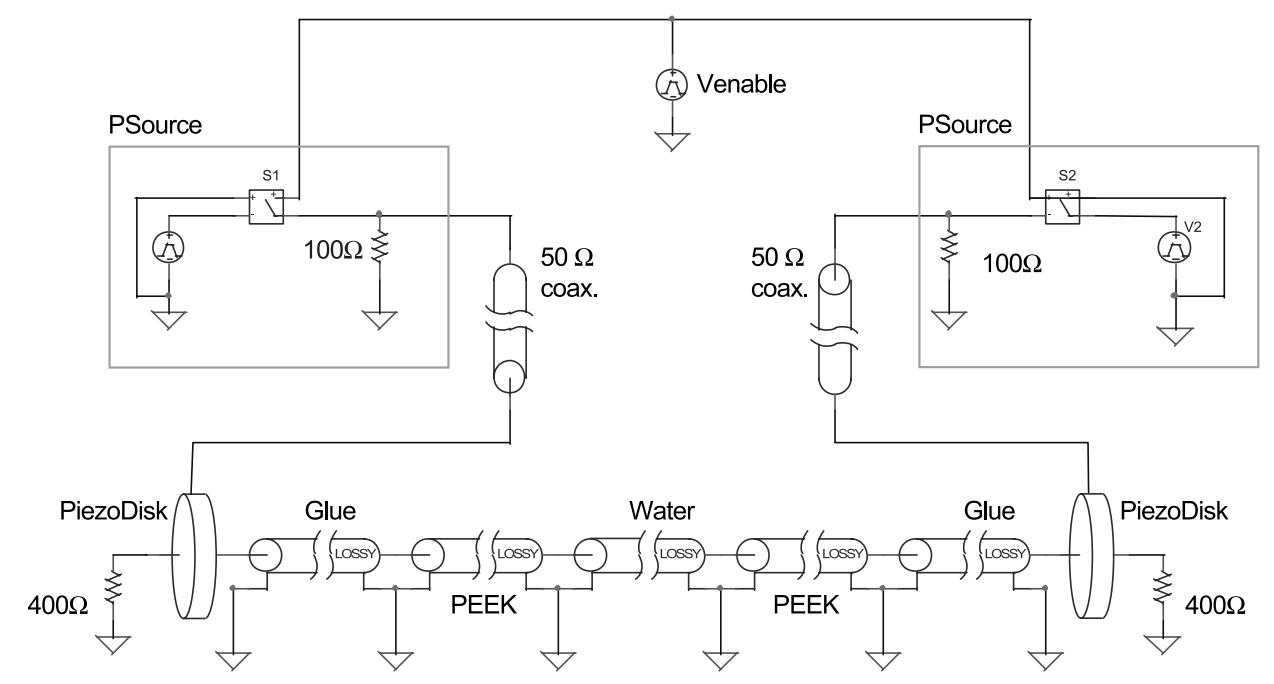

Fig. 2. Schematic representation of the SPICE model. 


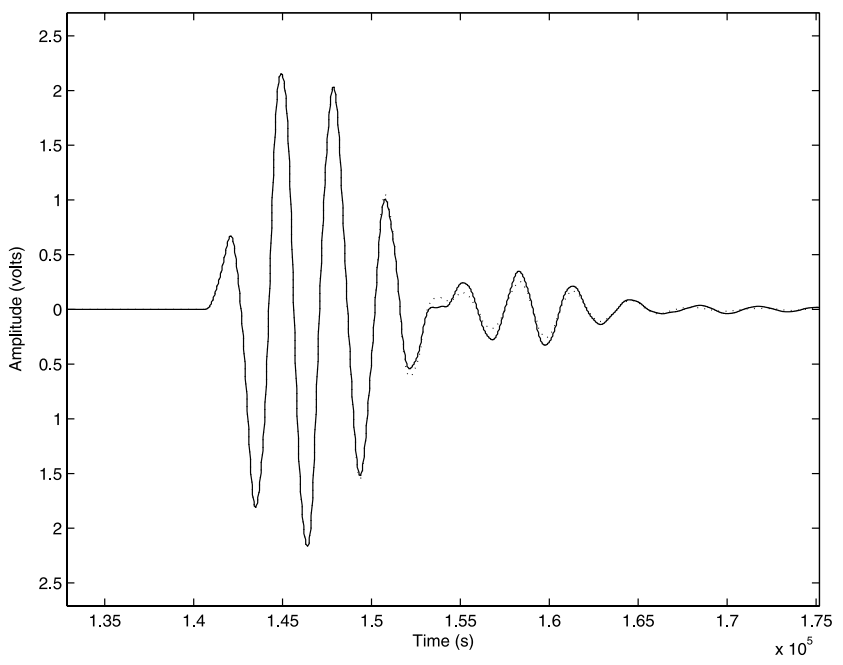

Fig. 3. The received pulses from a simulated flow meter.

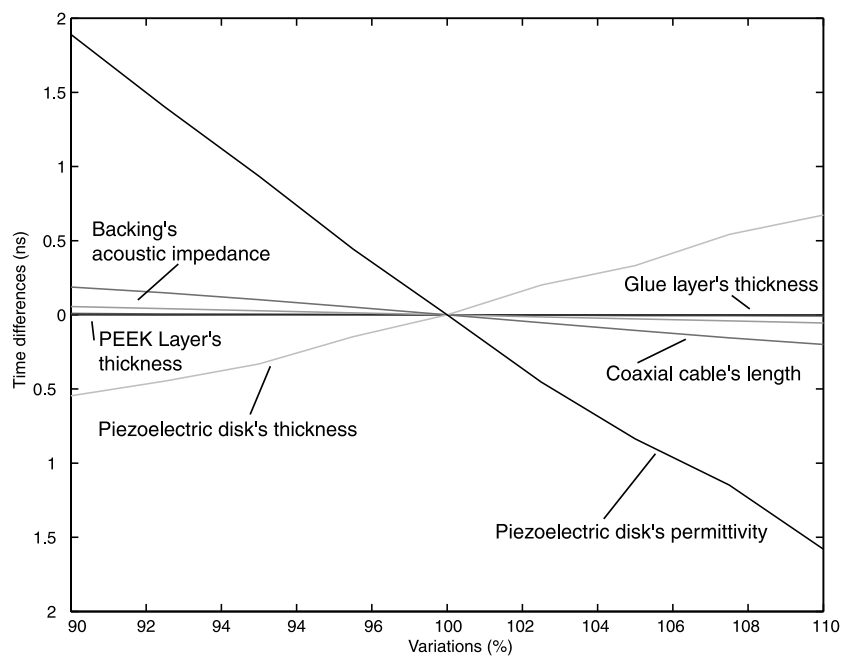

Fig. 4. Measured differences in times of flight due to non-identical transducers.

Table 1

Time differences in picoseconds due to variations in one transducer

\begin{tabular}{|c|c|c|c|c|c|c|c|c|c|}
\hline \multirow[t]{2}{*}{ Parameters } & \multicolumn{9}{|c|}{ Variations } \\
\hline & $-10.0 \%$ & $-7.5 \%$ & $-5.0 \%$ & $-2.5 \%$ & $-0.0 \%$ & $2.5 \%$ & $5.0 \%$ & $7.5 \%$ & $10.0 \%$ \\
\hline PEEK thickness & -0.25 & -0.97 & 0.16 & 0.19 & -1.13 & -0.14 & -0.22 & -0.20 & 0.21 \\
\hline Glue thickness & 9.8 & 5.6 & 4.8 & 2.8 & 0.0 & -1.5 & -6.0 & -6.0 & -7.7 \\
\hline $\begin{array}{l}\text { Backing acoustic } \\
\text { impedance }\end{array}$ & 56.0 & 41.6 & 27.6 & 13.6 & 0.0 & -13.9 & -27.5 & -41.1 & -54.8 \\
\hline Coaxial cable length & 187.9 & 148.4 & 101.7 & 52.1 & 0.0 & -53.0 & -105.1 & -155.1 & -199.8 \\
\hline Disk thickness & -546.1 & -446.7 & -330.1 & -148.0 & 0.0 & 200.3 & 332.3 & 542.6 & 673.1 \\
\hline Disk permittivity & 1890.5 & 1400.2 & 934.7 & 444.0 & 0.0 & -453.0 & -836.2 & -1147.5 & -1580.2 \\
\hline
\end{tabular}

\section{Conclusion}

Using simulations, we have shown that if the two ultrasonic transducers in a transit time flow meter are not identical then there is a time difference between the up- and downstream times of flights. Due to the different response of non-identical transducers, reciprocity across the transit time ultrasonic flow meter does not hold. Because it is only possible to manufacture identical transducers within a certain tolerance range, zero-flow calibration is necessary. A design strategy can be developed to counter the influence of a property variation by sizing another accordingly.

Future works are clearly laid out. First, the simulation results need to be validated with actual experiments. The actual electrical properties of paired transducers can be measured using a network analyzer. With those values and the measured dimensions of the transducers in the model, simulation results will be compared to measurements. In a second stage, the research will focus on ways to minimize this need for a zero calibration.

\section{References}

[1] L.C. Lynnworth, Ultrasonic Measurements for Process Control: Theory, Techniques, Applications, Academic Press, Inc, Boston, MA, 1989.

[2] J. Hemp, Theory of transit time ultrasonic flowmeters, Journal of Sound and Vibration 84 (1) (1982) 133-147.

[3] M. Willatzen, Ultrasound transducer modeling - General theory and applications to ultrasound reciprocal systems, IEEE Trans. Ultrason., Ferroelec., Freq. Contr. 48 (1) (2001) 100-112.

[4] A.D. Pierce, in: Acoustics: An Introduction to its Physical Principles and Applications, Acoustical Society of America, Woodbury, NY, 1989, p. 195 (Section 4.9).

[5] J. van Deventer, T. Löfqvist, J. Delsing, PSpice simulations of ultrasonic systems, IEEE Trans. Ultrason., Ferroelec., Freq. Contr. 47 (4) (2000) 1014-1024.

[6] W.M. Leach Jr., Controlled-sources analogous circuits and SPICE models for piezoelectric transducers, IEEE Trans. Ultrason., Ferroelec., Freq. Contr. 41 (1) (1994) 60-66.

[7] J. Johansson, Effects of parasitic components on ultrasound measurement system-PSpice simulations and measurements, IEEE Ultrason. Symp., Atlanta GA, submitted for publication.

[8] A. Hasting, The Art of Analog Layout, Prentice Hall, Upper Saddle River, NJ, 2001 